\title{
LIMITATIONS OF PEGYLATED NANOCARRIERS: UNFAVOURABLE PHYSICOCHEMICAL PROPERTIES, BIODISTRIBUTION PATTERNS AND CELLULAR AND SUBCELLULAR FATES
}

\author{
AYA AHMED SEBAK ${ }^{*}$ \\ Pharmaceutical Technology Department, Faculty of Pharmacy and Biotechnology, German University in Cairo (GUC). Main Entrance of El- \\ Tagamoa Al-Khames, New Cairo City, Egypt \\ Email: aya.sebak-mohamed@guc.edu.eg
}

Received: 27 May 2018, Revised and Accepted: 26 Jun 2018

\begin{abstract}
Assuming that polyethylene glycol (PEG)-conjugated or PEGylated nanocarriers always offer outstanding physicochemical properties and pharmacokinetics profiles when compared to non-PEGylated ones, is not always accurate. For example, drug-loaded PEGylated nanocarriers for the treatment of cancer will not magically escape the reticuloendothelial system (RES) sequestration and clearance, benefit from the enhanced permeability and retention (EPR) effect of the tumor leaky vasculature and preferentially accumulate in the target tissue or cells. This is too good to be true. In this review, several drawbacks of PEGylation will be discussed; for example, how PEGylation can give rise to unfavourable physicochemical characteristics (e. g. particle size and release patterns) and post in vivo administration limitations of the formulated nanocarriers (e. g. limited evasion of RES uptake, development of hypersensitivity reactions, reduced intracellular accumulation and interference with the subcellular processing of nanocarriers necessary to produce the intended pharmacological effect).
\end{abstract}

This review aims at providing better understanding of the pros and cons of PEGylation, encouraging the use of PEGylation with caution, avoiding the assumption that PEGylation will provide all advantages needed to deliver nanocarriers to the target tissue and looking for alternatives to optimize nanocarriers' utilization especially in the delivery of chemotherapeutic agents for the treatment of different types of cancer. This review comprises a summary of some of the reported literature between 2013 and 2018 using different search engines; PubMed, Science Direct and Google Scholar, and the keywords listed below.

Keywords: PEG, PEGylation, Nanoparticles, Nanovesicles, Drawbacks, Limitations, Alternatives

(C) 2018 The Authors. Published by Innovare Academic Sciences Pvt Ltd. This is an open access article under the CC BY license (http://creativecommons.org/licenses/by/4.0/) DOI: http://dx.doi.org/10.22159/ijap.2018v10i5.27568

\section{INTRODUCTION}

PEGylation, or the use of PEG, is one of the most common approaches utilized for improving nanocarriers' e. g. nanoparticles (NPs') or nanovesicles' (NVs') properties for in vivo administration. PEG, as a hydrophilic biocompatible stealth polymer, can decrease NPs sequestration by the RES organs (liver, spleen, kidneys). It prevents adsorption of proteins (opsonins) that tag the NPs or NVs to be engulfed by phagocytic cells in a process named opsonisation by providing steric hindrance preventing their interaction with macrophages necessary for the process of phagocytosis [1-5].

A closer insight to the mechanism by which PEG extends circulation half-life revealed that PEG could preferentially bind to other proteins that function as dysopsonins, which are proteins capable of preventing opsonisation or other protein fragments in place of opsonins. In one study, PEGylated lipoplexes have shown the higher extent of serum protein (but not opsonins) binding compared to non-PEGylated lipoplexes [6].

Also, PEG is a non-ionic (neutral) polymer that can mask surface charge and develop a near-zero zeta potential which eventually reduces non-specific interaction with macrophages and favour long circulation time in blood [7-10]. While highly charged, positive or negative, or hydrophobic NPs were found to attract complement proteins and be highly sequestered by RES organs. Positively charged NPs have even showed the highest extent of sequestration by RES organs; where they preferentially interact with negatively charged plasma proteins leading to higher RES uptake $[9,11]$.

Therefore, PEG can increase blood circulation time and reduce sequestration in the organs of the RES making NPs more available for uptake by their target organs or tissues e. g. tumor tissues [1-4, 12]. In other words, long-circulating nanocarriers have higher chance to benefit from the EPR promoted by the tumor leaky vasculature $[4,13-15]$.

Another interesting advantage for the PEGylated nanocarriers is their high ability to cross the blood-brain barrier (BBB) and accumulate in brain tissue more effectively than non-PEGylated ones possibly by transcytosis or receptor-mediated endocytosis mechanisms which need further to be elaborated. Diffusion through the brain parenchyma has also turned out to be a function of PEG coating density; i.e. the higher the PEG coating density, the higher the brain parenchymal diffusion $[4,16-$ 19]. In this regard, PEGylated nanocarriers have shown enhanced accumulation in pathological conditions characterized by BBB hyperpermeability e. g. gliosarcoma and multiple sclerosis (MS)-induced inflammation and brain tumors-induced EPR effect [16].

For these advantages, PEG has been regarded as a gold standard polymer in various applications and could not be neglected; however, on the other hand, many drawbacks have been reported over the years that could limit its applications (fig. 1);

\section{Major hurdles of PEGylation}

PEGylation that has been widely used to improve the physical and chemical properties of nanocarriers showed unfavourable profiles of an abrupt release of the loaded cargos before or right after in vivo administration, even before NPs sufficiently accumulate in target tissues.

Also, particle size enlargement has been observed on comparison of PEGylated and non-PEGylated nanocarriers which produced a reversed effect on the intended RES evasion and accumulation in tumor tissue by the EPR effect [6].

Deeper analysis of the effect of PEGylation on the cellular and subcellular fate showed reduced uptake and interference with NPs processing which could hinder the optimized pharmacological effect of the loaded drug [13]. Also, there has been evidence of the occurrence of PEG-containing vacuoles in cells. Reports so far also show the potential risk of PEG itself being a non-biodegradable polymer that can produce an immune response or cause hypersensitivity where PEG could generate antibodies that can neutralize their efficacy upon repeated administration [6].

Also, PEG synthesis and purification are difficult processes that give rise to an inherent polydispersity of the polymer which eventually causes batch-to-batch variability [20]. 

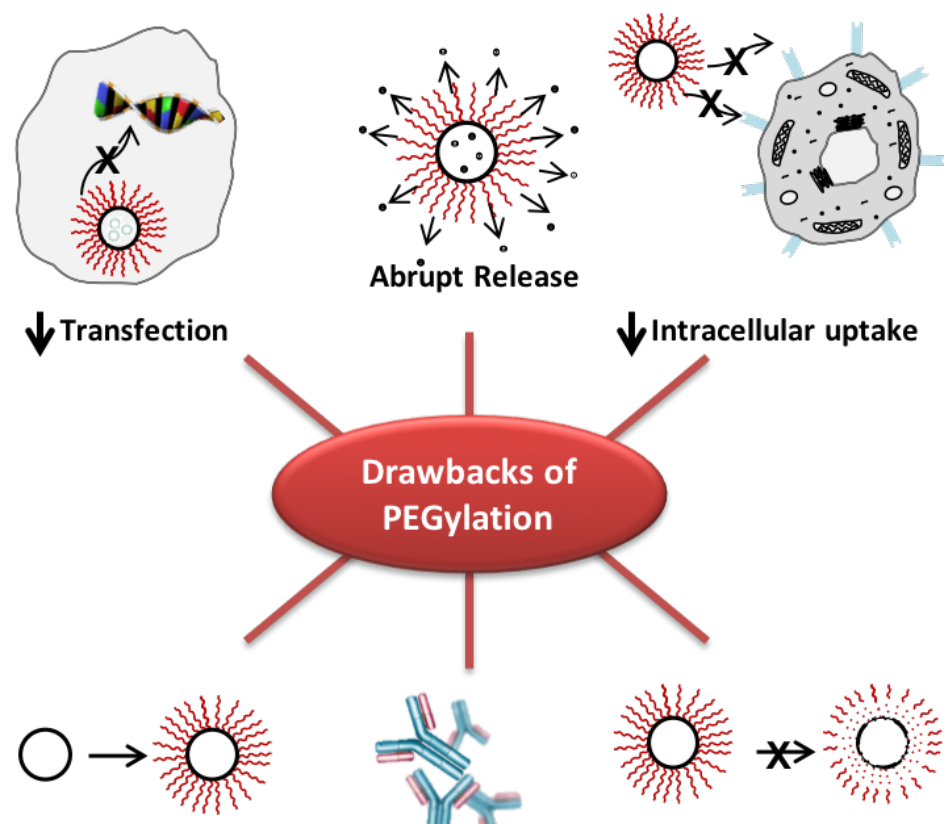

Size Enlargement

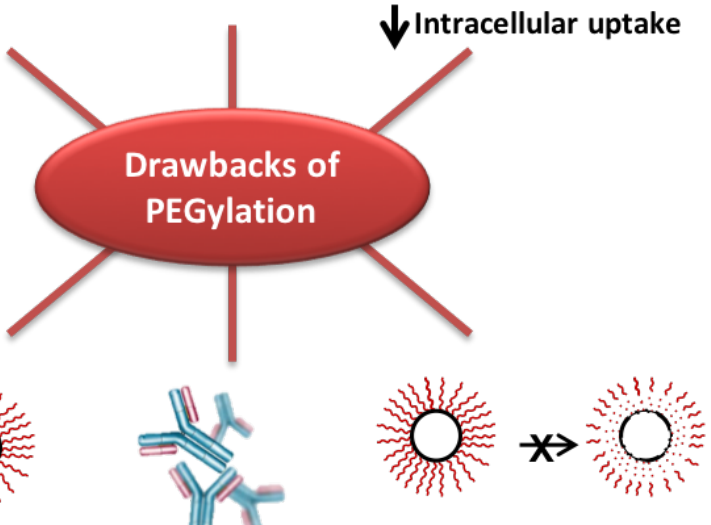

Non-Biodegradable

Anti-PEG antibody

Fig. 1: Main drawbacks of PEGylation of nanocarriers

\section{Unfavorable physicochemical properties of PEGylated nanocarriers}

PEGylation was reported to affect the physicochemical properties of NPs adversely causing enlargement of particle size-that could enhance RES uptake especially with large molecular weight PEG-and abrupt release of the drug loads from NPs over a short period of time. For example, PEGylated liposomes loaded with doxorubicin have shown a faster release of drug cargos than the non-PEGylated counterparts, where $90 \%$ of the loaded doxorubicin was released in $3 \mathrm{~h}$ post intravenous (IV) administration while the emptied liposomes were still circulating in blood before reaching the target site. This made the assumption that the loaded drug cargo is released after being up-taken by target cells not accurate. Similarly, the release of morphine from poly (L-lactide) or PLA-PEG block copolymer particles have shown to be much faster than release from PLA polymer ones. Also, the release rate has been shown to be a function of PEG percentage (\%) in the copolymer i.e. the initial burst release is more evident in particles with PEG content of 5\% compared to $3 \%$. While particles containing $0-1 \%$ of PEG showed no burst release, but rather a continuous sustained release over time. Upon estimating the $\mathrm{T}_{80 \%}$, which represents the time when the cumulative release percentage of morphine reached about $80 \%$, PEG-free particles showed around 15 and 30 times longer $\mathrm{T}_{80 \%}$ than particles containing 3 and 5\% of PEG respectively. This reflects that loaded cargos could be released from PEG-containing particles before degradation of the polymer. In other words, particles could become empty of the loaded cargos while they are still in circulation before their degradation. Moreover, a similar trend was reported for PEGylated and non-PEGylated poly (lactic-co-glycolic acid) (PLGA) and PLA NPs. Faster release profiles of hydrophilic drugs loaded in PEGylated NPs could be attributed to the hydrophilicity of PEG chains at the surface of the nanocarriers which act to attract water resulting in pronounced wetting/hydration of PEGylated NPs $[9,10,21,22]$.

RES evasion capacity not as expected or even worsened and reversed

As reported earlier in the advantages of PEGylation, the hydrophilic polymer provides steric hindrance and masking of surface charge that prevents adsorption of tagging proteins (opsonisation) which is a prerequisite of uptake by RES macrophages; however, PEGylation capacity of prevention of opsonisation has shown to be limited, and eventual opsonisation and RES clearance has been repeatedly reported. In one study, for example, temoporfin (photosensitizer used in photodynamic cancer therapy) was loaded in both PEGylated and non-PEGylated PLGA NPs and followed for in vivo behaviour. It has been reported that similar amounts of both NPs accumulated in the spleen and that the RES tissue distribution differed only slightly between PEGylated and non-PEGylated NPs showing inefficiency of PEGylation in RES evasion [23].

In another study, PEGylation of thiolated gelatin was observed to reduce mean residence time (MRT) and half-life of NPs in blood. In other words, the majority of NPs were cleared from the blood by circulating phagocytes and tissue macrophages (hepatic kupffer cells and macrophages in the spleen) and the NPs eventually accumulated in the liver and spleen [10]. A similar trend was reported for PEGylated and albumin-conjugated PEGylated liposomes, where results showed that albumin conjugation produced the longest circulation time [24]. Many factors control the effect of PEGylation on NPs' fate; these include the molecular weight, PEG polymer identity, composition and hydrophobicity, density and stability of the PEG in the coating layer, NP core properties, and repeated administration. These criteria if not properly controlled lead to unfavourable effects upon PEG coating [6, 13, 25-27].

The limited RES evasion capacity by PEGylation has been attributed to many phenomena; most importantly, size enlargement of nanocarriers, desorption of PEG from nanocarriers' surfaces and production of anti-PEG antibodies and development of an immune response that causes accelerated blood clearance (ABC) of PEGylated nanocarriers. These are explained in detail as follows;

\section{PEGylation causes enlargement of particle size}

Formulations possessing average particle size $\leq 150-200 \mathrm{~nm}$ were reported to be suitable for IV application [7, 28, 29]; being large enough to avoid filtration in the kidney, and small enough to avoid sequestration in the RES organs (liver, spleen, kidneys), and suitable for selective accumulation and retention in tumor by EPR effect [30-34].

In one study, PEGylation of PLGA NPs led to a larger particle size that had an effect on the pharmacokinetics of NPs and accounted for the abnormal decrease in the MRT of NPs in blood [10]. This means evasion of RES by PEG is not absolute. Larger size conferred by PEG 
layer could make them more accessible to RES organs than the smaller non-PEGylated PLGA NPs [9].

In another study, increasing the \% of PEG in PLGA NPs from $0 \%$ to $9 \%$ to $15 \%$ increased particle size from $\sim 740 \mathrm{~nm}$ to $1170 \mathrm{~nm}$ to $1995 \mathrm{~nm}$ respectively [35]. A similar observation was recorded in chitosan NPs; where PEGylated chitosan NPs possessed larger particle size $(\sim 290 \mathrm{~nm})$ than non-PEGylated ones $(\sim 200 \mathrm{~nm})[36]$. Also, PEG coating of nanostructured lipid carriers (NLCs) led to size enlargement from $(\sim 72 \mathrm{~nm})$ to $(\sim 92 \mathrm{~nm})[37]$.

\section{Insufficiency or desorption of PEG layer}

As reported earlier in the advantages of PEGylation, the hydrophilic polymer provides steric hindrance that prevents adsorption of tagging proteins (opsonisation) which is a prerequisite of uptake by RES macrophages; however, it has also been observed that PEG polymers could also desorb, leaving holes in surface coverage where opsonins can bind [38] or not provide full prevention of opsonisation at low density of PEG or using low molecular weight polymer. For example, with $5 \mathrm{~nm}$ gold NPs, the protective effect of PEG diminishes within hours [39].

\section{Immunogenicity of PEG}

PEG, the long-believed polymer to be non-antigenic could develop an immune response resulting in ABC of PEG-modified materials e. g. PEGylated proteins, liposomes, micelles, and biodegradable and nonbiodegradable nanocarriers. Where an anti-PEG antibody could be formed that results in the faster elimination of the second or further IV injected dose in animal studies and clinical settings [39]. This has been clarified by the formation of immune complexes which can be rapidly bound and cleared by macrophages via Fc receptor-mediated phagocytosis [40].

The $\mathrm{ABC}$ phenomenon is believed to result from anti-PEG IgM antibody produced by the spleen in response to a previouslyadministered dose. This type of antibody is, however, unable to solely promote sequestration by RES macrophages. However, these antibodies were hypothesized to bind to the second injected dose of PEG conjugates and form a complex with other serum components eventually promoting sequestration by RES $[6,13]$. For instance, the second injection of PEGylated liposomes witnessed reduced circulation time and increased hepatic and splenic accumulation in comparison to the first injected dose of the same formulation in the same animal [6]. Moreover, anti-PEG antibodies of both IgG and IgM isotypes were found in $25 \%$ of 250 healthy blood donors. This extraordinary evidence of anti-PEG antibodies was explained by the increasing exposure of the general population to PEG in different cosmeceutical and pharmaceutical products for many years [41]. Other reports show the incidence of anti-PEG antibodies in the range of $0.4 \%$ to $36 \%$ in naïve individuals [40].

In another study, a more severe adverse effect to the immunogenicity of PEG, hypersensitivity, has been observed clinically where three cases $(0.002 \%$ of the treated patients with advanced coronary artery disease) administered a commercial PEGcontaining contrast agent, SonoVue, developed life-threatening anaphylactic shock $[6,15,42]$.

The loss of activity of PEGylated pharmaceutical products upon repeated injection has also extended to anticancer therapeutics. PEGylated asparaginase is an integral part of therapy for acute lymphoblastic leukaemia (ALL) that is administered either intravenously (IV) or intramuscularly (IM). In a study, $46 \%$ of patients were reported to develop anti-PEG antibodies in response to IV administration that resulted in accelerated clearance of subsequent doses eventually causing silent inactivation of the treatment. In another study, $25 \%$ of patients were identified with pre-existing anti-PEG antibodies prior to treatment that could be attributed to their pre-exposure to PEG from other pharmaceutical or cosmeceutical products that are widely available. Another study showed the development of anti-PEG antibodies after IM administration of the PEGylated asparaginase [13, 43, 44].

Therefore, it could be concluded that PEG effect does not last for a long time, and eventually, opsonisation and clearance by RES organs still occur [45].
The prevalence of anti-PEG antibodies has shown to be higher in females than males; this could be explained by their higher exposure to PEG-containing products e. g. skin lotions, deodorant sticks, hair care products and face makeup products or their higher tendencies to have autoimmune responses [40].

\section{Unfavourable cellular and subcellular fate}

Another major drawback of PEGylation is the reduced intracellular uptake of the PEGylated NPs in comparison to non-PEGylated ones due to the steric hindrance caused by the projecting chains of PEG polymers and the hydrophilicity of NPs' surfaces as well. In other words, neutral and hydrophilic NPs e. g. PEG-modified NPs show slower and lower internalization in target cells than both of positively and negatively charged ones. For example, intracellular uptake of PEGylated NPs was noted to drop to one-third of the uptake of non-PEGylated ones [46, 47]. Also, PEGylation of folic acid (FA)-targeted dendrimers has been also reported decreasing the tumour-specific targeting by FA [20].

Therefore, even that PEGylation could sometimes evade sequestration of drug-loaded nanocarriers by RES cells and increase the probability of extravasation of NPs through the tumor leaky vasculature, it reduces interaction with target tissues e. g. tumor cells, whereby PEG shields internalization by non-immune cells by both steric hindrance and masking of NPs surface charge required for optimum cellular uptake [13].

In a similar manner, the interesting properties of PEG to enhance BBB crossing encouraged the application of PEGylated nanocarriers for brain delivery; however, it should not be neglected that there is another barrier that could hinder the successful delivery of therapeutic agents, which is the microglial cells that constitute the immune component of the central nervous system (CNS) itself. These cells are characterised by high phagocytic activity that resembles RES macrophages that could compete with other non-immune neural cell types for NPs' uptake. Jenkis et al., 2016 have been concerned with comparison of NPs' uptake in their PEGylated and non-PEGylated forms in different neural cell types; their findings confirmed that PEGylation reduces NPs' uptake in all cell types; immune and non-immune neural cells suggesting that PEGylation evades non-specific cell uptake that could outweigh its advantage of BBB and parenchymal diffusion when applied for brain drug delivery [16].

The effect of PEGylation on the target cells is not limited to reduced uptake but extends to inhibition of endosomal escape, which results in significant loss of activity of the delivery systems e. g. gene and nucleic acid delivery systems for cancer treatment where the NPs' cargos are degraded in the digestive acidic medium of the endolysosomal system [41].

The detrimental effect of PEGylation extends also to the interference with the intracellular processing of NPs required for optimum release of loaded cargos to achieve the intended goals. For example, PEGylated nanocarriers intended for gene and siRNA delivery have shown reduced transfection and silencing rates that could not be explained solely by the previously discussed reduced uptake caused by PEGylation. Therefore, it was concluded that PEGylation affects the intracellular fate of nanocarriers after being internalized by target cells. In different studies, PEGylation levels as low as 0.4-0.5\% showed a drastic decrease in transfection rates indicating that the therapeutic gene expression in the target cells may be significantly compromised by PEGylation [13].

\section{Non-biodegradability of PEG polymers}

Despite that PEG has been the gold standard in pharmaceuticals' application for many years possessing the longest clinical track record amongst many other polymers and that it has been widely used as a biocompatible polymer, it should be noted that it is nonbiodegradable and that its ultimate fate and potential side effects that could arise from its accumulation need further to be elaborated. Also, there are concerns about its bioaccumulation in lysosomes of healthy tissue. Therefore, limiting the molecular weight should be taken into account for in vivo application [13, 48-50]. 


\section{Strategies to overcome PEGylation drawbacks}

\section{Proper selection of PEG polymer and conjugation chemistry}

There are many available approaches to overcome the PEG dilemma (fig. 2), for example, choosing appropriate polymers with less or no antigenicity and controlling the different criteria that can affect PEGylation outcomes e. g. PEG molecular weight, density and stability of the PEG coating $[6,25,27]$. In one study, branched PEG was shown to be the least immunogenic and antigenic when compared to methoxy-PEG (mPEG) and other un-branched PEG forms [6]. Also, a more recent study by Shimizu et al. showed that
hydroxyl-PEG could also serve as a potential alternative to MPEG. It could attenuate the elicitation of the ABC phenomenon when conjugated to PEGylated liposomes by the production of less antiPEG IgM antibodies [51]. In another report, the immunogenicity of different PEG derivatives showed the following order; t-butoxy-PEG (tBu-PEG)>mPEG>hydroxyl-PEG (OH-PEG). Also, two types of antiPEG antibodies have been proposed; methoxy-specific and backbone-specific. Where OH-PEG was found to produce antibodies that have a similar affinity to both $\mathrm{MPEG}$ and OH-PEG, while mPEG antibodies recognize $\mathrm{mPEG}$ more effectively than OH-PEG [26].

PEG Type and Conjugation Chemistry (Type of linker or coating density)

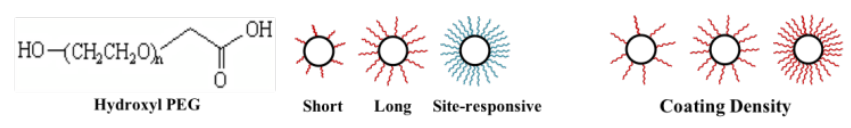

Alternative Coatings
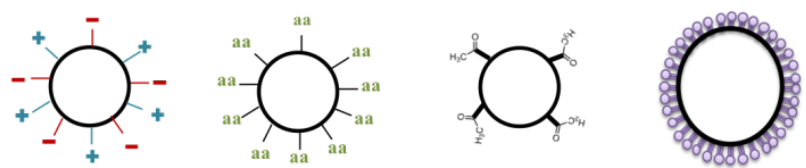

Zwitterionic coatin

Amino acid coating

Acetylation
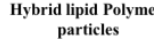

Markers of Self

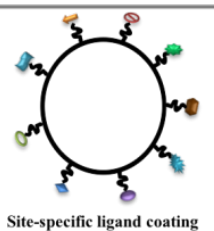

Fig. 2: Alternative strategies to overcome PEGylation drawbacks

Also, the issue of non-biodegradability of PEG could be addressed and solved; for example, by conjugating PEG to degradable PLA or poly (glycolic acid), (PGA) segments to form corresponding biodegradable copolymer $[52,53]$.

In the meanwhile, attempting to overcome the detrimental effect of PEGylation on gene and siRNA delivery, PEG has been conjugated to shorter lipid anchors to facilitate loaded cargos diffusion out of the lipid-based delivery systems. In another attempt, PEG was attached to site-specific cleavable linkers e. g. matrix metalloprotease (MMP)sensitive linkers in tumors or $\mathrm{pH}$ sensitive linkers to be cleaved by the low $\mathrm{pH}$ conditions encountered after cellular uptake in the lysosomal degradation pathway [13].

\section{Alternative coatings}

In another approach to overcome PEGylation dilemma, alternative coatings of nanocarriers have been studied. For example, NPs with zwitterionic coatings were found to be able to evade brain immune components and reduce the rate of non-specific binding for proteins and lipids without steric hindrance [16].

Moreover, 5-nm gold NPs were coated with amino acids such as lysine or cysteine to constitute a mixed-charge monolayer. This coating could prevent protein adsorption in fetal bovine serum [39, 54].

Also, another study employed acetylation of dendrimers as an alternative to PEGylation. This approach offered the advantage of RES evasion without adding steric hindrance that could reduce the interaction between FA on the acetylated dendrimers and folate receptor on the surface of the target cells that could reduce the uptake of the NPs as in the case of FA-conjugated PEGylated dendrimers. The mechanism by which acetylation reduces RES clearance was hypothesized to occur by neutralizing the cationic surface of the dendrimers and reducing the electrostatic interactions with serum proteins that are required for uptake by RES macrophages. Acetylation is also potentially applicable for modification of other types of nanocarriers and possesses an added advantage of better control over the size and polydispersity of the NP compared to PEGylation [20].

Utilization of hyperbranched polyglycerol (HPG) as an alternative to the typical PEG has shown to be promising in the brain drug delivery. HPG showed more favourable physicochemical properties e. g. higher drug loading, higher NPs' surface coverage and higher effectiveness for ligand conjugation and more prolonged circulation time [50,55]. HPG coating imparted excellent stability of magnetic $\mathrm{Fe}_{3} \mathrm{O}_{4} \mathrm{NPs}$ in cell culture medium. These coated NPs were nontoxic to mammalian cells and showed minimal uptake by macrophages. HPG also offers a platform for staging the NPs surfaces for active targeting [49].

Other stealth polymers such as chitosan, poly (carboxybetaine), polysaccharides, polypeptides, poly (vinyl alcohol) and poly (2oxaline) have been also investigated $[25,26]$.

Lipids also can be used as coatings for NPs to increase their blood circulation time as an alternative to PEG, producing hybrid lipidpolymer NPs. Hybrid NPs represent a promising drug delivery platform that has shown controllable particle size, surface functionality, high drug loading yield, triggered or stimuliresponsive drug release profile, excellent in vitro and in vivo stability, biocompatibility and biodegradability. Also, co-loading of drugs with different physicochemical properties became possible. In other words, the polymeric core functions to encapsulate either water or oil-soluble drugs and to provide robust structures, whereas the external lipid coat offers the following advantages; (i) acts as a biocompatible shield, (ii) a template for surface modifications, (iii) a 
barrier preventing the fast leakage of water-soluble drugs (iv) possesses favourable stability in serum (longer circulation times) and (v) good cellular accumulation and targeting ability [33, 56-61]. In another study, it was observed that the incorporation of phospholipid into polymeric NPs could significantly enhance their intracellular accumulation [62].

\section{Utilization of markers of self; target-specific ligands}

In order to address the problem of reduced intracellular uptake by target cells upon PEGylation, markers of self or target-specific ligands e. g. FA or transferrin can be attached to the surface of the NPs in order to achieve active targeting (enhance cellular uptake into target tissues and to avoid exposure of healthy cells) and also help minimize or avoid complement activation [39]. Arginine-glycine-aspartic acid (RGD) tripeptide has also shown to improve the selectivity, binding and uptake of the NPs by targeted cells because of its ability to be recognized by integrin receptors that are ovreexpressed on tumor cells. This strategy can specifically enhance the limited uptake drawback due to steric hindrance by PEG polymers [41]. In another study, targeted PEGylated liposomes produced by conjugation to the fibronectin-mimetic peptide, PR_b, to target $\alpha_{5} \beta_{1}$ in metastatic colon cancer, have shown better transfection efficiency than non-targeted ones [5]. Further optimization of PEGylated liposomes was performed by dual targeting; in a study, PR_b and AG86 peptides were used to target $\alpha_{5} \beta_{1}$ and $\alpha_{6} \beta_{4}$ respectively were conjugated with different ratios to the PEGylated liposomes and investigated for their internalization and transfection efficiencies in dual receptor expressing cancer cells. Results showed high selectivity of the employed nanocarrier system especially in ovarian cancer cells (SKOV-3) characterized by an almost equal expression of the two receptors [63].

The same targeting strategy could be applied to optimize the brain delivery of drugs in PEGylated nanocarriers. For example, PEGylated gold NPs, when functionalized with transferrin-specific peptide, showed significantly higher accumulation in brain tumor [64]. Also, PEGylated PLGA NPs or polymersomes when conjugated with phage display peptide or lactoferrin respectively showed higher brain accumulation $[64,65]$. Dual targeting has also been employed to optimize brain drug delivery; where paclitaxcel was conjugated to cyclo-[Arg-Gly-Asp-D-Phe-Lys] peptide which can interact specifically with integrin receptors, overexpressed on glioma cells. This conjugate was further loaded into PEGylated lipid-polymer hybrid NPs [66].

\section{CONCLUSION}

In conclusion, PEG has shown, for decades, the outstanding potential for enhancement of properties of drug delivery systems that made its application in pharmaceuticals and cosmeceuticals widespread. However, improper control of the PEGylation process gave rise to many drawbacks that render its application limited. Also, chronic exposure to PEG for many years made the immune system of humans and animals alert to the molecule producing antibodies; that limit the efficacy of the administered drug in most of the times and could give rise to more serious side effects e. g. anaphylactic shock. Therefore, PEGylation should be used with great caution. Also, looking for alternatives has emerged to become an ultimate necessity.

\section{AUTHORS CONTRIBUTIONS}

All the work have been carried out by me

\section{CONFLICT OF INTERESTS}

The author reports no declarations of interest

\section{REFERENCES}

1. Kudgus RA, Walden CA, Mcgovern RM, Reid JM, Robertson JD, Mukherjee P. Tuning pharmacokinetics and biodistribution of a targeted drug delivery system through the incorporation of a passive targeting component. Sci Rep 2014;4:5669.

2. Lázaro I, Haddad S, Sacca S, Orellana Tavra C, Fairen Jimenez D, Forgan RS. Selective surface PEGylation of UiO-66 nanoparticles for enhanced stability, cell uptake, and $\mathrm{pH}-$ responsive drug delivery. Chem 2017;2:561-78.
3. Barnard AS. Heterogeneous PEGylation of diamond nanoparticles. Nanoscale 2017;9:70-4.

4. Suk JS, Xu Q, Kim N, Hanes J, Ensign LM. PEGylation as a strategy for improving nanoparticle-based drug and gene delivery. Adv Drug Delivery Rev 2016;99:28-51.

5. Shaji J, Menon I. Recent advances in nanocarrier-based therapeutic and diagnostic tools for colorectal cancer. Int J Curr Pharm Res 2015;7:9-16.

6. Zhang F, Liu M, Wan H. Discussion about several potential drawbacks of PEGylated therapeutic proteins. Biol Pharm Bull 2014;37:335-9.

7. Sadat SM, Jahan ST, Haddadi A. Effects of size and surface charge of polymeric nanoparticles on in vitro and in vivo applications. J Biomater Nanobiotechnol 2016;7:91-108.

8. Mohammad AK, Reineke JJ. Quantitative detection of PLGA nanoparticle degradation in tissues following intravenous administration. Mol Biol Cell 2013;10:2183-9.

9. Rafiei P, Haddadi A. Docetaxel-loaded PLGA and PLGA-PEG nanoparticles for intravenous application: pharmacokinetics and biodistribution profile. Int J Nanomed 2017;12:935-47.

10. Xu J, Gattacceca F, Amiji M. Biodistribution and pharmacokinetics of EGFR-targeted thiolated gelatin nanoparticles following systemic administration in pancreatic tumour-bearing mice. Mol Pharm 2013;10:2031-44.

11. Behzadi S, Serpooshan V, Tao W, Hamaly MA, Alkawareek MY, Dreaden EC, et al. Cellular uptake of nanoparticles: a journey inside the cell. Chem Soc Rev 2017;46:4218-44.

12. Chen S, Yang K, Tuguntaev RG, Mozhi A, Zhang J, Wang PC, et al. Targeting tumor microenvironment with PEG-based amphiphilic nanoparticles to overcome chemoresistance. Nanomedicine 2016;12:269-86.

13. Verhoef JJF, Anchordoquy TJ. Questioning the use of PEGylation for drug delivery. Drug Delivery Transl Res 2013;3:499-503.

14. Kobayashi H, Watanabe R, Choyke PL. Improving conventional enhanced permeability and retention (EPR) effects; what is the appropriate target? Theranostics 2014;4:81-9.

15. D'souza AA, Shegokar R. Polyethylene glycol (PEG): a versatile polymer for pharmaceutical applications. Expert Opin Drug Delivery 2016;13:1257-75.

16. Jenkins SI, Weinberg D, Al-Shakli AF, Fernandes AR, Yiu HHP, Telling ND, et al."Stealth" nanoparticles evade neural-immune cells but also evade major brain cell populations: implications for PEGbased neurotherapeutics. J Controlled Release 2016;224:136-45.

17. Saraiva C, Praça C, Ferreira R, Santos T, Ferreira L, Bernardino L. Nanoparticle-mediated brain drug delivery: overcoming blood-brain barrier to treat neurodegenerative diseases. J Controlled Release 2016;235:34-47.

18. Tamba BI, Streinu V, Foltea G, Neagu AN, Dodi G, Zlei M, et al. Tailored surface silica nanoparticles for blood-brain barrier penetration: preparation and in vivo investigation. Arab J Chem 2018. https://doi.org/10.1016/j.arabjc.2018.03.019

19. Xu Q, Ensign LM, Boylan NJ, Schön A, Gong X, Yang JC, et al. Impact of surface polyethylene glycol (PEG) density on biodegradable nanoparticle transport in mucus ex vivo and distribution in vivo. ACS Nano 2015;9:9217-27.

20. Rattan R, Bhattacharjee S, Zong H, Swain C, Siddiqui MA, Visovatti $\mathrm{SH}$, et al. Nanoparticle-macrophage interactions: a balance between clearance and cell-specific targeting. Bioorg Med Chem 2017;25:4487-96.

21. Yang X, Wu S, Wang Y, Li Y, Chang D, Luo Y, et al. Evaluation of self-assembled HCPT-loaded PEG-b-PLA nanoparticles by comparing with HCPT-loaded PLA nanoparticles. Nanoscale Res Lett 2014;9:1-8.

22. Chen F, Yin G, Liao X, Yang Y, Huang Z, Gu J. Preparation, characterization and in vitro release properties of morphineloaded PLLA-PEG-PLLA microparticles via solution enhanced dispersion by supercritical fluids. J Mater Sci Mater Med 2013;24:1693-705.

23. Voon SH, Tiew SX, Kue CS, Lee HB, Kiew LV, Misran M, et al. Chitosan-coated poly(lactic-co-glycolic acid)-diiodinated boron-dipyrromethene nanoparticles improve tumor selectivity and stealth properties in photodynamic cancer therapy. J Biomed Nanotechnol 2016;12:1431-52. 
24. Perche F, Torchilin VP. Recent trends in multifunctional liposomal nanocarriers for enhanced tumor targeting. J Drug Delivery 2013;201:3705265.

25. Yang Q, Lai SK. Anti-PEG immunity: emergence, characteristics, and unaddressed questions. Wiley Interdiscip Rev Nanomed Nanobiotechnol 2015;7:655-77.

26. Zhang P, Sun F, Liu S, Jiang S. Anti-PEG antibodies in the clinic: current issues and beyond PEGylation. J Controlled Release 2016;244:184-93.

27. Wan X, Zhang J, Yu W, Shen L, Ji S, Hu T. Effect of protein immunogenicity and PEG size and branching on the anti-PEG immune response to PEGylated proteins. Process Biochem 2017;52:183-91.

28. Dhand C, Prabhakaran MP, Beuerman RW, Lakshminarayanan $\mathrm{R}$, Dwivedi N, Ramakrishna S. Role of the size of drug delivery carriers for pulmonary and intravenous administration with an emphasis on cancer therapeutics and lung-targeted drug delivery. RSC Adv 2014;4:32673-89.

29. Hoshyar N, Gray S, Han H, Bao G. The effect of nanoparticle size on in vivo pharmacokinetics and cellular interaction. Nanomedicine (Lond) 2016;11:673-92.

30. Hickey JW, Santos JL, Williford JM, Mao HQ. Control of polymeric nanoparticle size to improve therapeutic delivery. J Controlled Release 2015;219:536-47.

31. Shalgunov V, Zaytseva-zotova D, Zintchenko A, Levada T, Shilov $\mathrm{Y}$, Andreyev D, et al. Comprehensive study of the drug delivery properties of poly (L-lactide)-poly (ethylene glycol) nanoparticles in rats and tumour-bearing mice. J Controlled Release 2017;261:31-42.

32. Ray S, Mishra A, Mandal TK, Sa B, Chakraborty J. Optimization of the process parameters for the fabrication of a polymer coated layered double hydroxide-methotrexate nanohybrid for the possible treatment of osteosarcoma. RSC Adv 2015;5:102574-92.

33. Voigt J, Christensen J, Shastri VP. Differential uptake of nanoparticles by endothelial cells through polyelectrolytes with affinity for caveolae. Proc Natl Acad Sci 2014;111:2942-7.

34. Yoo W, Yoo D, Hong E, Jung E, Go Y, Singh SVB, et al. Acidactivatable oxidative stress-inducing polysaccharide nanoparticles for anticancer therapy. J Controlled Release 2018;269:235-44.

35. Moayedian T, Mosaffa F, Khameneh B, Tafaghodi M. Combined effects of PEGylation and particle size on the uptake of PLGA particles by macrophage cells. Nanomed J 2015;2:299-304.

36. Kaur S, Vinay C, Narang R, Geeta A. Comparative mucopenetration ability of metronidazole-loaded chitosan and PEGylated chitosan nanoparticles. Asian J Pharm Clin Res 2017;10:125-30.

37. Srichaivatana K, Ounaroon A, Tiyaboonchai W. Development and characterization of piper retrofractum extract loaded mucoadhesive nanostructured lipid carriers for topical oral drug delivery. Int J Pharm Pharm Sci 2017;9:79-86.

38. Jahan ST, Sadat SM, Walliser M, Haddadi A. Targeted therapeutic nanoparticles: an immense promise to fight against cancer. J Drug Delivery 2017:9090325. https://doi.org/ $10.1155 / 2017 / 9090325$

39. Kettiger H, Schipanski A, Wick P, Huwyler J. Engineered nanomaterial uptake and tissue distribution: from cell to organism. Int J Nanomed 2013;8:3255-69.

40. Chen BM, Su YC, Chang CJ, Burnouf PA, Chuang KH, Chen $\mathrm{CH}$, et al. Measurement of pre-existing IgG and IgM antibodies against polyethylene glycol in healthy individuals. Anal Chem 2016;88:10661-6.

41. Hatakeyama H, Akita H, Harashima H. The polyethyleneglycol dilemma: advantage and disadvantage of PEGylation of liposomes for systemic genes and nucleic acids delivery to tumors. Biol Pharm Bull 2013;36:892-9.

42. Seicean A, Jinga M. Harmonic contrast-enhanced endoscopic ultrasound fine-needle aspiration: fact or fiction? Endosc Ultrasound 2017;6:31-6.

43. van der Sluis IM, Vrooman LM, Pieters R, Baruchel A, Escherich G, Goulden N, et al. Consensus expert recommendations for identification and management of asparaginase hypersensitivity and silent inactivation. Haematologica 2016;101:279-85.
44. Browne E, Moore C, Lu Z, Sykes A, Jeha S, Mandrell BN. Clinical characteristics of intravenous PEG-asparaginase hypersensitivity reactions in patients undergoing treatment for acute lymphoblastic leukemia. J Pediatr Oncol Nurs 2016;137:10160-3.

45. Dubey SK, Samanta MK, Dubey SK, Mishra P. Monoclonal antibody conjugated nanoparticles targeted to prostate tumor cells. Indian J Pharm Biol Res 2014;2:21-9.

46. Mao Z, Zhou X, Gao C. Influence of structure and properties of colloidal biomaterials on cellular uptake and cell functions. Biomater Sci 2013;1:896.

47. Oh N, Park JH. Endocytosis and exocytosis of nanoparticles in mammalian cells. Int J Nanomed 2014;9:51-63.

48. Qi Y, Chilkoti A. Protein-polymer conjugation-moving beyond PEGylation. Curr Opin Chem Biol 2015;28:181-93.

49. Hadjesfandiari N, Parambath A. Stealth coatings for nanoparticles. In: Parambath A. Engineering of Biomaterialfor Drug Delivery Systems: Beyond Polyethylene Glycol. Elsevier; 2018. p. 345-61.

50. Lowe S, O'Brien-Simpson NM, Connal LA. Antibiofouling polymer interfaces: poly(ethylene glycol) and other promising candidates. Polym Chem 2015;6:198-212.

51. Shimizu T, Abu Lila AS, Fujita R, Awata M, Kawanishi M, Hashimoto $\mathrm{Y}$, et al. A hydroxyl PEG version of PEGylated liposomes and its impact on anti-PEG IgM induction and on the accelerated clearance of PEGylated liposomes. Eur J Pharm Biopharm 2018;127:142-9.

52. Saez Martinez V, Olalde B, Martinez Redondo D, Braceras I, Morin F, Valero J, et al. Degradable poly(ethylene glycol)-based hydrogels: synthesis, physicochemical properties and in vitro characterization. J Bioact Compat Polym 2014;29:270-83.

53. Ulbricht J, Jordan R, Luxenhofer R. On the biodegradability of polyethylene glycol, polypeptoids and poly(2-oxazoline)s. Biomaterials 2014;35:4848-61.

54. Murthy AK, Stover RJ, Hardin WG, Schramm R, Nie GD, Gourisankar $\mathrm{S}$, et al. Charged gold nanoparticles with essentially zero serum protein adsorption in undiluted fetal bovine serum. J Am Chem Soc 2013;135:7799-802.

55. Zhou Y, Peng Z, Seven ES, Leblanc RM. Crossing the blood-brain barrier with nanoparticles. J Controlled Release 2018;270:290303.

56. Garg NK, Tandel N, Jadon RS, Tyagi RK, Katare OP. Lipidpolymer hybrid nanocarrier-mediated cancer therapeutics: current status and future directions. Drug Discovery Today 2018. Doi:10.1016/j.drudis.2018.05.033

57. Dong $\mathrm{W}$, Wang $\mathrm{X}$, Liu $\mathrm{C}$, Zhang $\mathrm{X}$, Zhang $\mathrm{X}$, Chen $\mathrm{X}$, et al. Chitosan-based polymer-lipid hybrid nanoparticles for oral delivery of enoxaparin. Int J Pharm 2018;547:499-505.

58. Bose RJC, Ravikumar R, Karuppagounder V, Bennet D, Rangasamy S, Thandavarayan RA. Lipid-polymer hybrid nanoparticle-mediated therapeutics delivery: advances and challenges. Drug Discovery Today 2017;22:1258-65.

59. Date T, Nimbalkar V, Kamat J, Mittal A, Mahato RI, Chitkara D. Lipid-polymer hybrid nanocarriers for delivering cancer therapeutics. J Controlled Release 2018;271:60-73.

60. D'Souza AA, Shegokar R. Polymer: lipid hybrid nanostructures in cancer drug delivery: successes and limitations. In: Holban AM, Grumezescu A. Nanoarchitectonics for smart delivery and drug targeting. Elsevier; 2016. p. 431-63.

61. Li Q Xia D, Tao J, Shen A, He Y, Gan Y, et al. Self-assembled coreshell-type lipid-polymer hybrid nanoparticles: intracellular trafficking and relevance for oral absorption. J Pharm Sci 2017;106:3120-30.

62. Salatin S, Khosroushahi AY. Overviews on the cellular uptake mechanism of polysaccharide colloidal nanoparticles. J Cell Mol Med 2017;20:1-19.

63. Levine RM, Kokkoli E. Dual-ligand $\alpha 5 \beta 1$ and $\alpha 6 \beta 4$ integrin targeting enhances gene delivery and selectivity to cancer cells. J Controlled Release 2017;10:24-36.

64. Sun C, Ding Y, Zhou L, Shi D, Sun L, Webster TJ, et al. Noninvasive nanoparticle strategies for brain tumor targeting. Nanomedicine 2017;13:2605-21.

65. Li J, Zhang C, Li J, Fan L, Jiang X, Chen J, et al. Brain delivery of NAP with PEG-PLGA nanoparticles modified with phage display peptides. Pharm Res 2013;30:1813-23. 
66. Agrawal U, Chashoo G, Sharma PR, Kumar A, Saxena AK, Vyas SP. Tailored polymer-lipid hybrid nanoparticles for the delivery of drug conjugate: Dual strategy for brain targeting Colloids Surf B 2015;12:414-25. 